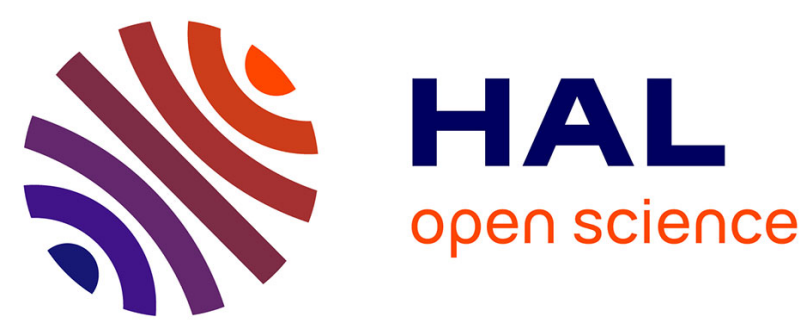

\title{
Performance Analysis of an Enhanced Spectrally Aware RF front end Receiver under Non-linearity
}

\author{
Nicolas Grollier, Sébastien Houcke, Michaël Pelissier
}

\section{To cite this version:}

Nicolas Grollier, Sébastien Houcke, Michaël Pelissier. Performance Analysis of an Enhanced Spectrally Aware RF front end Receiver under Non-linearity. 10th International Symposium on Signal, Image, Video and Communications (ISIVC), Apr 2021, Saint Etienne (en ligne), France. 10.1109/ISIVC49222.2021.9487534 . hal-03331349

HAL Id: hal-03331349

https://imt-atlantique.hal.science/hal-03331349

Submitted on 1 Sep 2021

HAL is a multi-disciplinary open access archive for the deposit and dissemination of scientific research documents, whether they are published or not. The documents may come from teaching and research institutions in France or abroad, or from public or private research centers.
L'archive ouverte pluridisciplinaire HAL, est destinée au dépôt et à la diffusion de documents scientifiques de niveau recherche, publiés ou non, émanant des établissements d'enseignement et de recherche français ou étrangers, des laboratoires publics ou privés. 


\title{
Performance Analysis of an Enhanced Spectrally Aware RF front end Receiver under Non-linearity
}

\author{
Nicolas Grollier ${ }^{1}$, Sébastien Houcke ${ }^{1}$, Michaël Pelissier ${ }^{2}$ \\ ${ }^{1}$ Lab-STICC, CNRS, IMT Atlantique, 29238 Brest, France \\ Email: \{nicolas.grollier,sebastien.houcke\}@imt-atlantique.fr \\ ${ }^{2}$ CEA - LETI, University Grenoble Alpes, F-38000 Grenoble, France \\ Email: michael.pelissier@cea.fr
}

\begin{abstract}
In most of $\mathrm{RF}$ receivers ( $\mathrm{RX})$ the analog chain aims to improve the overall Signal to Interference plus Noise Ratio (SINR) by filtering out interferences in the Signal Of Interest (SOI) vicinity. However, cognitive radio applications require a high dynamic range $\mathrm{RX}$, pushing a high constraint on the analog section. In particular, nonlinearities may damage the overall SINR and Bit Error Rate (BER) due to blocking and intermodulation processes in presence of strong interference. $R X$ linearization state of the art methods that rely on the feedforward concept have shown the RX linearization improvement on the BER. Nonetheless, benefits of other linearization methods such as the feedback loop concept, are unstudied. In this article, our aim is to decrease the power of unwanted harmonics thanks to a feedback loop. We first explain the variable gain enhancement concept. Then, we present an algorithm that dynamically adapts the RX parameters (gains and ADC margins) to make it work in a linear regime. We analyze the effect of such a method in terms of BER. Important BER improvement is noticed in blocking and saturating situations due to co-channel interferers.
\end{abstract}

\section{INTRODUCTION}

In this article we consider the context of Professional Mobile Radios (PMR) radios. The FITNESS project (see [1]) aims to prepare the future of PMR in Europe and in the world. New functionalities requested by missioncritical markets are added, while preserving backwards compatibility with existing narrowband PMR systems. The considered receiver is narrowband, and has stringent specifications to make it compliant with the four European PMR norms in terms of receiver selectivity.

Several PMR communications channels close to the signal of interest (SOI) may be present at ADC level and $60 \mathrm{~dB}$ above the SOI. Commercial automatic gain control (AGC) are unable to deal with a dynamic up to $141 \mathrm{~dB}$ with channel bandwidth of $12.5 \mathrm{kHz}$. This specification is particularly severe. So, a specific architecture that allows amplification stage to be reconfigurable was defined. This architecture is meant to be fully integrated, and able to cope with tight linearity and gain requirements of a PMR receiver (RX) front-end. To ensure that ADCs will not clip the received signal, large back-off digitization margins have to be left. One other issue is caused by the nonlinear behavior of RX analog components. Indeed, amplifiers or mixers (see [2]) used outside of their linear region are responsible for intermodulation products harmonic and even gain blocking. Such terms lay into the SOI bandwidth, as developed in [3]. In the following, unwanted signals are referred as interferers.

The nonlinearity cancellation is a vast topic that is generally addressed in an analog way. This is usually a well-known designer problem, and examples of mechanisms are proposed in [2], [4] to limit such effects under specific bounds. Devices specifications could be adjusted by a proper analog design to maximize one or several criteria amongst power efficiency, linearity, peak to average power ratio or adjacent channel power ratio. Three main principles exist to compensate for nonlinearities in the analog domain: feedback, feedforward and predistortion.

Nonetheless, in this paper we consider the whole RX as a series of nonlinear blocks, instead of a single device (e.g. a power amplifier). We assume that there is no knowledge of the signal input, which at first discards the feedback and the predistortion methods. To our best knowledge, it appeared that all state of the art methods of RX linearization rely on the feedforward principle. Two main approaches are to be distinguished. On one hand, there are many fully digital techniques [3], [5][7]. In these papers, various ways allows the cancellation of almost all the power of Inter Modulation Distortion of order 3 (IMD3) harmonics. Their overall ideas are similar: modeling IMD3 harmonics and then subtract it from the received signal with an adaptive mechanism. On the other hand, a mixed analog-digital strategy was proposed in [8] and is refined in [9]. In theses papers IMD terms are generated thanks to an analog mechanism 
that model the nonlinear mechanism. This signal is digitized and subtracted from the amplified input signal thanks to a least mean square algorithm.

However, none of these methods allow to relax significantly ADCs dynamic range and sampling frequency requirements. Indeed, to be modeled, interferers have to be digitized with respect of Shannon's rule. In [10] we have already proposed a novel method to decrease IMD3 products power as a proof of concept. It showed that thanks to a sensing mechanism, the RX could be partially aware of its spectral environment and able to change its parameters (gains and ADC margins) to work in its linear region. The proposed sensing mechanism is able to detect nonlinear harmonics before the compression point and gain clipping.

In this article, we design a feedback loop to adjust the RX gain. We rely on the detection method [10] to detect the nonlinear harmonics presence. Our aim is to maintain the RX at the limit of detectable harmful harmonics. It allows to take advantage of the largest gain while decreasing the power of unwanted components. We analyze the effect of such a system on decoding performance, in blocking tests bench. In section II, we develop the nonlinear system model and remind the variable gain control concept. Section III is devoted to practical implementation issues and the simulation counterpart. Main detection mechanism principles are developed as well. To underline possible improvements obtained with our method, we show the BER improvement through simulations in section IV. Section V concludes the paper.

\section{System Model}

\section{A. Received Signal Model}

Let us define useful and interferer continuous baseband signal models in (1).

$$
z_{i}(t)=\beta_{i} \sum_{k=-\infty}^{\infty} a_{k, i} h\left(t-k T_{i}\right)
$$

Let $T_{i}$ be the symbol period, $a_{k, i}$ be the complex random symbols with unit variance, $h(t)$ be a continuous shaping filter (normalized in energy) and $\beta_{i}$ the square root power of $z_{i}(t)$. Here $z_{1}(t)$ is meant to be the useful signal and $z_{2}(t)$ the unwanted signal. In the following, only the $h(t)$ shape is known as a root raised cosine filter. We consider that the SOI and the interferer are independent from each other, and $a_{k, i}$ are independent MPSK symbols.

The signal $y_{i}(t)$ at carrier frequency $f_{c i}$ can be expressed as:

$$
y_{i}(t)=\Re\left[z_{i}(t) e^{\jmath 2 \pi f_{c i} t}\right]
$$

$y_{2}(t)$ parameters are assumed to be unknown except for its carrier frequency $f_{c 2}$. The composite received signal is the combination of SOI and interferer. This signal is defined in (3).

$$
x(t)=\sum_{i=1}^{2} y_{i}(t)
$$

\section{B. Nonlinear Model}

Our whole receiver is modeled as a memoryless polynomial expressed as:

$$
p(t)=\alpha_{1} x(t)+\alpha_{2} x(t)^{2}+\alpha_{3} x(t)^{3}+w(t)
$$

where $\alpha_{k}, k \in\{1,2,3\}$ are characteristics of the RX front-end and $w(t)$ is an additive white Gaussian noise. In the following, $\alpha_{2}$ is assumed zero since harmonics in $|x(t)|^{2}$ are easily discarded in a heterodyne architecture with proper filtering stages and a front-end differential structure. The nonlinear amplification coefficient $\alpha_{3}$ is obtained thanks to (5) (see [2]), where $V_{I I P 3}$ is the RX Input Interception Point of third order.

$$
\alpha_{3}=-\frac{4}{3} \frac{\alpha_{1}}{V_{I I P 3}^{2}}
$$

The substitution of (3) into (4) leads to the complete formula of harmonic creation for the nonlinear model. All terms are listed in [3], but $\alpha_{2}$ is not considered here. We focus on the received signal baseband representation in a given sub-band of interest. The SOI in this sub-band is given by:

$$
\begin{aligned}
p(t)= & \Re\left\{\left(\alpha_{1}+\frac{3}{4} \alpha_{3}\left|z_{1}(t)\right|^{2}\right.\right. \\
& \left.\left.+\frac{3}{2} \alpha_{3}\left|z_{2}(t)\right|^{2}\right) z_{1}(t) e^{j 2 \pi f_{c 1} t}\right\}+w(t)(6)
\end{aligned}
$$

We clearly see that in (6) the third right hand-side term depends on interferer's power. Hence, when the interference power comes too high, the overall linear gain $\alpha_{1}$ is blocked.

\section{Variable Gain Enhancement Concept}

In a conventional RF receiver, the dynamics of the useful signal is determined by compromising between linear amplification and the background noise (NF) added by the chain components. The presence of unwanted signals is also taken into account in this choice, by limiting amplification and setting additional digitization margins (e.g. PAPR). However, as presented in [10], having an $\mathrm{RX}$ that is aware of the presence of interferers allows the gain to be dynamically changed to remain in a linear regime despite the presence of unwanted signals. This method also reduces digitization margins by increasing the conversion dynamic. There are two different use scenarios:

1) Sensitivity: which reflects the presence of a lowpowered useful signal (there are no jammers, $\beta_{2}=0$ ). If there is no disturbance signal, the gain can be increased to reduce the background noise.

2) Linearity: which corresponds to two situations: a) the useful signal is the only signal present but has a high power; b) the wanted signal and at least one high power interferer are present. In (6), these cases correspond to a gain blocking situation due 
to the third order term of (4). Thus, the gain should be reduced to allow a better linearity, but at the cost of a degradation of the NF.

\section{VARIABle GAin IMPlEMENTATION ASPECTS}

In this section we first develop the gain adaptation issue in a real RX. Then, we go on with main principles of the chosen spectrum sensing method. Finally, we explain the practical method we used in simulation.

\section{A. Gain Adaptation Mechanisms}

For several cascaded nonlinear stages, the circuit nonlinearity is described by a relation equivalent to the well known Friis formula. From [2], we have:

$$
\frac{1}{V_{I I P 3, t o t}^{2}} \approx \frac{1}{V_{I I P 3,1}^{2}}+\frac{\alpha_{1,1}^{2}}{V_{I I P 3,2}^{2}}+\ldots+\frac{\prod_{k=1}^{n-1} \alpha_{1, k}^{2}}{V_{I I P 3, n}^{2}}
$$

An analysis of this equation is useful to underline mathematically what we propose to do. The (7) is the general expression for $n$ cascaded nonlinear stages. Let define $\alpha_{1, i}$ the linear gain of stage $i$ and $V_{I I P 3, i}$ their corresponding IIP3, with $i \in\{1, \ldots, n\}$. From (5), we recall that $V_{I I P 3, i}$ coefficients are linked to (4) by the nonlinear gain $\alpha_{3}$ such as:

$$
\alpha_{3, i}=-\frac{4}{3} \frac{\alpha_{1, i}}{V_{I I P 3, i}^{2}}
$$

(8) is a relation that can be applied indifferently for each stage $i$ or for the overall cascaded stage. Hence, any modification on a particular analog component impacts all the following RX chain.

In particular, if we consider the linearity scenario: the linear gain has to be decreased. A short analysis of (7) shows that when coefficients $\alpha_{1, i}$ decrease, so do the quantity $1 / V_{I I P 3, t o t}^{2}$. In (8), when $V_{I I P 3_{i}}$ increases the nonlinear gain $\alpha_{3, i}$ decreases. So, increasing $V_{I I P 3, t o t}$ makes the circuit more linear, which is the searched effect in the linearity scenario. However, the maximum gain decrease is limited by the noise floor and the Friis formula in (9). For $m$ stages [2] indicates that:

$N F_{\text {tot }}=1+\left(N F_{1}-1\right)+\frac{N F_{2}-1}{\alpha_{1,1}}+\ldots+\frac{N F_{m}-1}{\prod_{k=1}^{m-1} \alpha_{1, k}}$

Hence, a gain decrease makes the noise floor increases. In practice the gain can be decreased until the noise floor becomes too high. This approach is also valid in the sensing scenario. In the two cases, the minimum affordable gain makes the noise floor too important. On the contrary, the maximum amplification makes the circuit too nonlinear.

In an implementation point of view, the gain of each $\mathrm{RX}$ components is not tunable. It means that, one has to adapt specific gains coefficients in order to obtain the desired amplification. Moreover, changing the gain of a unique component makes the RX characteristic different.
In the following we assume that $\alpha_{1, t o t}$ and $V_{I I P 3, t o t}$ are continuous and continuously modifiable.

Furthermore, to realize computer simulations a relation between $\alpha_{1, t o t}$ and $\alpha_{3, t o t}$ has to be modeled. In the aim to change the linear gain parameter, one has to change in accordance with the $V_{I I P 3, t o t}$ value. Nonetheless, as seen in (7) $V_{I I P 3, t o t}$ is a function of the linear gain such as:

$$
\left(V_{I I P 3, t o t}\right)_{\mid d B}=f\left(\left(\alpha_{1, t o t}\right)_{\mid d B}\right)
$$

This relation is useful only in a simulation context. Indeed, in a real RX the gain is adapted to the needs and the nonlinear amplification is a parameter controlled by the chosen linear gain value. So, updating the $V_{I I P 3, t o t}$ value is not necessary.

\section{B. Detection of nonlinear harmonics}

To make the receiver aware of its spectral environment, we must identify whether the RX is working in its non-linear region, and why. The presence of an unwanted signal and/or an excess of power of the wanted signal creates many harmonics whose expressions are detailed in [3]. We wish to use the presence of these harmonics to detect the non-linear operation of the RX. We based our work on the criterion developed in [11], which requires few symbols and also allows a quick adaptation of the gain. In the following, we refer to this method as the Cyclostationary Nonlinear Harmonics Detector (CNHD). The idea is to detect the most powerful harmonics that are located at $f_{c 2}, 2 f_{c 1}$ and $2 f_{c 2}$. They correspond to the carrier frequency of the unwanted signal and the frequencies of the non-linear harmonics due to the wanted signal power and the interfering signal.

\section{Practical Implementation}

Let us now develop the feedback loop to adjust automatically the RX gain. The considered scenario is the linearity scenario, which means that a powerful interferer is present. We propose the scheme represented in fig.1. In this figure, the CNHD bloc stands for Cyclostationary Nonlinear Harmonics Detector (see section III.B). Let us define the input signal vector $\underline{x}_{N}(k)$ as the block of $N$ samples such as $\underline{\boldsymbol{x}}_{\boldsymbol{N}}(\boldsymbol{k})=[x(k N), \ldots, x(k N+$ $N-1)] . N$ is considered large enough to perform the detection with confidence. Let also define $\underline{\Phi}_{N}(k)=$ $\left[\underline{\boldsymbol{x}}_{\boldsymbol{N}}(\boldsymbol{k}) ; \underline{\boldsymbol{x}}_{\boldsymbol{N}}^{\mathbf{3}}(\boldsymbol{k})\right]$ a $2 \times N$ matrix, $\boldsymbol{W}=\left[\alpha_{1}, \alpha_{3}\right]$ a vector of gain and $\underline{\boldsymbol{w}}_{N}(\boldsymbol{k})=[w(k N), \ldots, w(k N+N-1)]$ a $1 \times N$ complex AGWN samples vector such as $\underline{p}_{N}(\boldsymbol{k})$ is defined as in (11) (equivalent to (4)).

$$
\underline{p}_{N}(k)=W \underline{\Phi}_{N}(k)+\underline{w}_{N}(k)
$$

For each bloc of $N$ samples the CNHD estimates $\hat{J}_{N, L}\left(4 f_{c 2}\right)$, the detection criterion. If $\hat{J}_{N, L}\left(4 f_{c 2}\right)$ is greater than $\Gamma$ (the detection threshold), it decides that the saturation is due to the interferer presence. So, the 


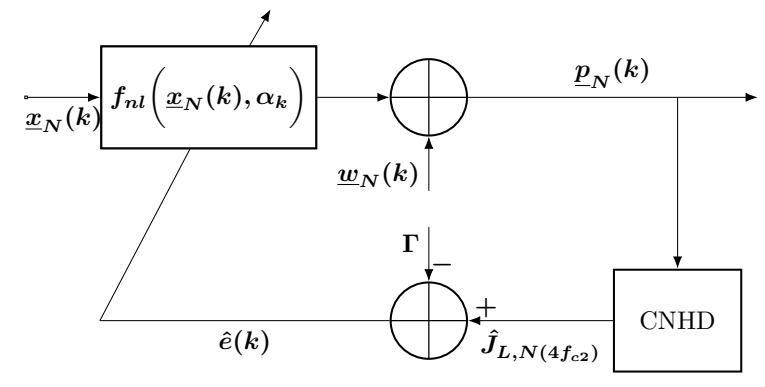

Fig. 1. Adaptive Feedback Loop with a Cyclostationary Nonlinear Harmonics Detector (CNHD)

distance of $\hat{J}_{N, L}\left(4 f_{c 2}\right)$ to $\Gamma$ gives us an indication on the power of this unwanted harmonic. Let define the estimated error $\hat{e}(k)$ for the block $k$ in (12).

$$
\hat{e}(k)=\hat{J}_{N, L}\left(4 f_{c 2}\right)-\Gamma
$$

This formulation is equivalent to maintaining the $\mathrm{RX}$ at the limit of detectable harmful harmonics. It allows to take advantage of the largest affordable gain while decreasing the power of unwanted components. However, one can notice that the chosen method is not adaptive filtering. Indeed, original samples of $\underline{x}_{N}(k)$ before its processing through RX analog components remain unknown. Hence, a minimization of the quadratic error in the Wiener sense can not be done.

The best way to adjust the gain value is to realize a gradient descent minimizing the quadratic error $\hat{e}(k)^{2}$. It requires to assume that $\hat{e}(k)^{2}$ is convex, which implies that $\hat{J}_{N, L}\left(4 f_{c 2}\right)$ depends on $\alpha_{1}$ and is monotone. The ideal gradient descent updating relation is:

$$
\hat{\alpha}_{1, t o t}(k+1)_{\mid d B}=\hat{\alpha}_{1, t o t}(k)_{\mid d B}-\mu \frac{\partial \hat{e}(k)^{2}}{\partial \alpha_{1}}
$$

Here $\mu$ is a step size that is chosen in function of the algorithm complexity. Indeed, it can be constant, function of $k$ or optimal depending on the needs. One can notice that the gradient descent in (13) implies to use the theoretical expression of $\hat{J}_{N, L}\left(4 f_{c 2}\right)$ as a function of $\alpha_{1, t o t}$.

As we lack such an expression, we propose an empirical solution. We propose to use (12) as it represents the distance to the ideal gain value, to adjust the amplification of the whole circuit. This approach is valid since the value of $\hat{J}_{N, L}\left(4 f_{c 2}\right)$ is linked to the gain thanks to (4). So, we propose an approximated solution inspired from the NLMS algorithm that takes into account the value of $\hat{e}(k)$ in (14).

$$
\begin{aligned}
\hat{\alpha}_{1, t o t}(k+1)_{\mid d B}= & \hat{\alpha}_{1, t o t}(k)_{\mid d B}-\mu(k) \operatorname{sgn}(\hat{e}(k)) . \\
& \log _{10}(\operatorname{sgn}(\hat{e}(k)) \hat{e}(k)+1)(14)
\end{aligned}
$$

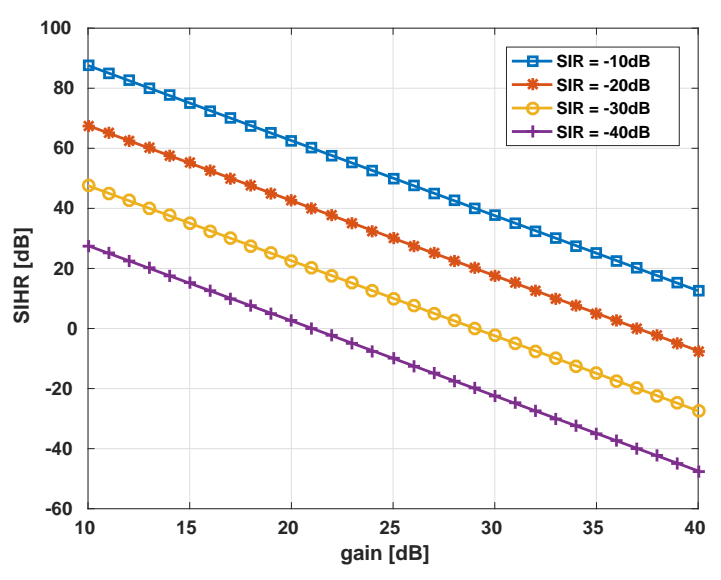

Fig. 2. Comparison of theoretical power of in-band components for several SIR values as a function of the RX Gain

Here, $\operatorname{sgn}($.$) stands for the sign function and \mu(k)=$ $10 / k$ is a variable step size that allows a convergence to a fixed value. Then, the $\hat{V}_{I I P 3, t o t}$ value has to be updated with the relation (10) to update the nonlinear model.

Now, two remarks can be made:

- The first point is that $\hat{e}(k)$ directly depends on $\Gamma$, which is set to satisfy a particular false alarm probability $\left(P_{f a}\right)$. So, changing the false alarm probability directly impacts the estimated gain $\hat{\alpha}_{1}(k+1)$. When we consider (14), one can observe that decreasing $P_{f a}$ means less detection. So it leads to decrease $\hat{\alpha}_{1}(k+1)$ less frequently and still working in a nonlinear regime.

- The second point is that (14) is not optimal in the Wiener sense. There is no guaranty that the relations converge to a value that minimizes the MMSE problem. Hence, we have to check if the final value actually decreases the nonlinear harmonic power.

To answer these two points, we propose to examine the power of the nonlinear term that lays in the SOI bandwidth.

We saw in (6) that the theoretical expression of the blocking term, denoted as $s(t)$, is: $s(t)=$ $3 / 2 \alpha_{3} z_{1}(t)\left|z_{2}(t)\right|^{2}$. From this relation, $s(t)$ theoretical power is developed in (15).

$$
P_{s}=E\left[|s(t)|^{2}\right]=\left(\frac{3}{2} \alpha_{3} \beta_{1} \beta_{2}^{2}\right)^{2}
$$

(15) is used to define a new comparison metric between the power of $s(t)$ and the power of $\alpha_{1} y_{1}(t)$ (the ideally amplified SOI): the Signal to In-band Harmonic Ratio (SIHR).

$$
S I H R=\frac{P_{\alpha_{1} y_{1}}}{P_{s}}
$$

This definition is different from the SIR, which is the ratio $\beta_{1} / \beta_{2}$ (SOI power over interferer power). The relation in (16) is represented graphically in fig.2. The 


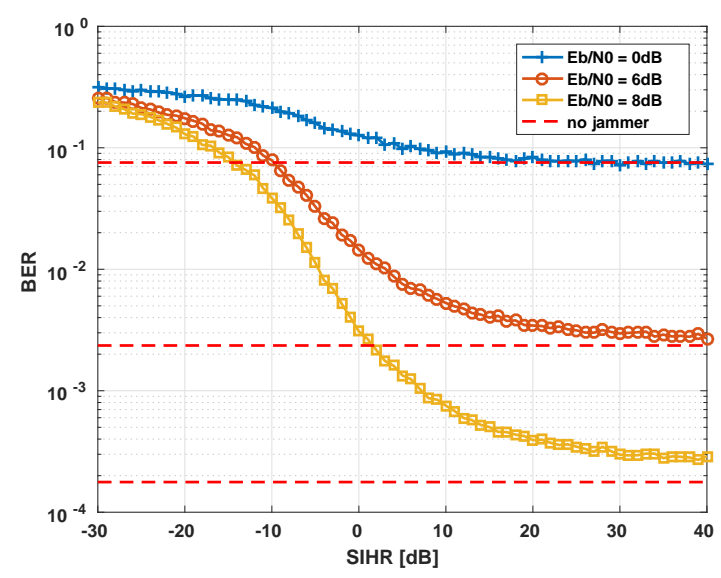

Fig. 3. Influence of SIHR on the BER for several $E_{b} / N_{0}$ values at fixed $\mathrm{SIR}=-30 \mathrm{~dB}$

first noticeable point is the linear relation between the SIHR and gain values. Let us now study the $-30 \mathrm{~dB}$ SIR curve as an example. Considering $\alpha_{1, \text { tot }}$ equals $32 \mathrm{~dB}$, the corresponding SIHR is $-11.4 \mathrm{~dB}$. It means that the in-band harmonic power is much higher than the SOI power. For now, we assume that a SIHR close to $20 \mathrm{~dB}$ implies that the in band unwanted harmonic has no influence on the decoding process. This assumption will be verified thanks to simulations and the fig. 3 analysis in the next section. So, one can use fig. 2 to check the best value of $\alpha_{1}$ for each SIR values. That way, for a $-30 \mathrm{~dB}$ SIR the gain has to be set at $21 \mathrm{~dB}$. Knowing the nonlinear gain, signal and interferers powers, this method gives a gain value without any adaptive algorithm. However, in this application, we have no access to such informations.

In tab.I, we sum-up several values of gain after a few algorithm iterations. According to this table, in the previous example a $21 \mathrm{~dB}$ gain is obtained for a $P_{f a}$ set at $5 \%$.

\section{Simulation and Numerical Results}

\section{A. Experimental Conditions}

To respect the Shannon's condition for the interferer signal, we set a sampling rate $f_{s}=10 f_{c 1}$. The shaping filter $h(t)$ is defined as a square-root raised cosine of period $T=2.5 T_{1}$, a roll-off at 0.8 and span at 6 symbols. Blocker and useful signal are in adjacent channels, and the interferer carrier frequency is defined

\begin{tabular}{|l|l|l|l|l|l|l|}
\hline \multirow{2}{*}{$\boldsymbol{\alpha}_{\mathbf{1}}(\boldsymbol{\infty})[\mathrm{dB}]$} & \multicolumn{7}{|c|}{$\boldsymbol{P}_{\boldsymbol{f a}}[\%]$} \\
\cline { 2 - 7 } & $\mathbf{5 0}$ & $\mathbf{2 0}$ & $\mathbf{1 0}$ & $\mathbf{5}$ & $\mathbf{1}$ & $\mathbf{0 . 1}$ \\
\hline SNR $=\infty[\mathrm{dB}]$ & 12.6 & 17.2 & 20.0 & 21.0 & 22.2 & 23.9 \\
\hline
\end{tabular}

TABLE I

GAIN VALUES AFTER ALGORITHM CONVERGENCE FOR SEVERAL FALSE ALARM PROBABILITY, SIR $=-30 \mathrm{~dB}, E_{b} / N_{0}=\infty$

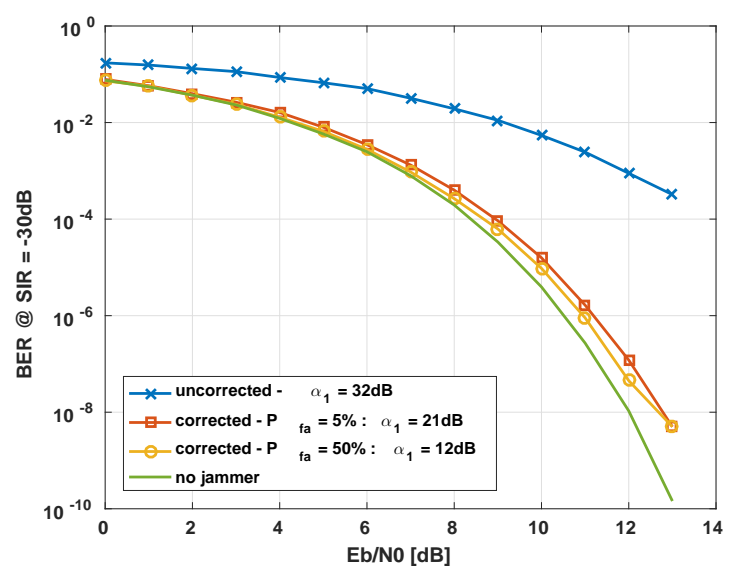

Fig. 4. Comparison of BER curves for uncorrected and corrected RX gain, for a $\mathrm{SIR}=-30 \mathrm{~dB}$

as $T_{2}=T_{1} / 2.4$. Both SOI and interferer are 4PSK modulated, with independent and identically distributed symbols. As discussed in [11], we assume that 8 symbols are enough to perform a detection of such signals using the CNHD method. We defined the SOI power $\beta_{1}$ to $-106.0 \mathrm{dBm}$. The detection threshold $\Gamma$ is fixed for a $P_{f a}$ of $5 \%$, as it represents a good tradeoff between detection and gain adjustment.

The RX gain is initialized at $\alpha_{1, t o t}(1)=32 d B$, when the corresponding $V_{I I P 3, t o t}(1)=-12.7 \mathrm{dBm}$. For different $\alpha_{1, t o t}(k)$ values the relation in (10) has to be developed. The $V_{I I P 3, t o t}$ value can be measured for a given $\alpha_{1, t o t}$ thanks to a two-tone test. For the particular RX front-end we considered, we tested several linear gain values and performed a two-tone test for each one. That way, we obtained an experimental relation between $\alpha_{1, t o t}$ and $V_{I I P 3, t o t}$.

$$
\left(V_{I I P 3, t o t}(k)\right)_{\mid d B}=-\left(\left(\alpha_{1, t o t}(k)\right)_{\mid d B}+10.7\right)
$$

Nonlinear model coefficients are then adapted thanks to relations (14) and (17).

\section{B. Simulation Results}

Let us first consider the assumption that a SIHR close to $20 \mathrm{~dB}$ is enough to have a BER close to the "no interferer" case. To make sure that the unwanted harmonic power in the SOI band is small compared to the SOI power, we provide fig.3. For several noise levels, we measured the influence of the SIHR value. We see that for each $E_{b} / N_{0}$ level, a negative SIHR (dB) implies a degraded BER. When the SIHR comes positive, the BER improves to get closer of its value when no interferer is present. One can see that the BER decreases to a stable value when the SIHR increases. So, past a SIHR of approximately $20 \mathrm{~dB}$, even a larger gain decrease may not lead to a significant BER improvement. This behavior is verified in the following simulations. 


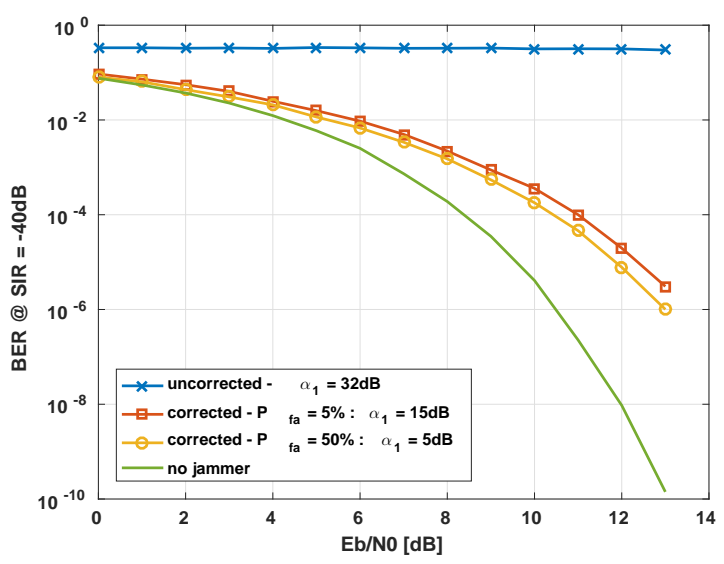

Fig. 5. Comparison of BER curves for uncorrected and corrected RX gain, for a SIR $=-40 \mathrm{~dB}$

Let now tackle the effect of the gain adaptation through a BER analysis. In fig.4, we defined a SIR such as the RX is faintly nonlinear (i.e. $-30 \mathrm{~dB}$ ). As it can be seen, the uncorrected BER is severely degraded: for a $-10^{3}$ BER value, there is a $5 \mathrm{~dB} E_{b} / N_{0}$ gap when compared to the "no interferer" case. Nonetheless, the RX is still able to decode a part of the received signal as the curve decreases when the $E_{b} / N_{0}$ ratio increases. It means that the unwanted harmonic in the SOI bandwidth is powerful, but not dominant. However, our gain adjustment mechanism allows a significant BER improvement. We represent several BER curves for which $\alpha_{1}$ is different. In compliance with the SIHR analysis, the BER is improved by a gain decrease, while the SIHR is smaller than $20 \mathrm{~dB}$. For a BER of $10^{-4}$, the improvement is close to BER when no interferer is present. Nonetheless, there is indeed a small difference between the $21 \mathrm{~dB}$ and the $12 \mathrm{~dB}$ BER curves. If we compare now the $21 \mathrm{~dB}$ BER curve with the no interferer case, for a $10^{-7}$ BER the improvement margin is about $0.85 \mathrm{~dB}$, which is small. Whatever the gain, there is still a small gap between the theoretical bound and gain adapted BER curves.

Finally, let us assume a SIR equals $-40 \mathrm{~dB}$ such as the RX works in a highly nonlinear regime. Such a powerful interferer makes the RX saturates, as shown in fig.5. We clearly see that without the gain adaptation mechanism, the BER is constant even when the noise power decreases. Our method allows the BER to be significantly improved. When compared with the $5 \mathrm{~dB}$ gain BER curve, the gap is very small, accordingly with fig. 3 remarks. However, as the interferer is very powerful (even after the adapted filter), its contribution is too important to be removed completely. That is why a large gap exists between the corrected gain BER curve and the theoretical bound.

\section{CONCLUSION}

In this work, we first recalled the method principles to make the RX works in its linear region thanks to a feedback loop. Based on results in [10], [11] we proposed an adaptive mechanism to maintain nonlinear harmonics power small. To our best knowledge, this concept is not tackled yet in the state of the art. Then, we analyzed the effects of a gain adaptation on in-band IP3 harmonics. This analysis allows to confirm that a gain maximization is possible while minimizing the nonlinear harmonics power. Important BER improvement is noticed in blocking and saturating situation. This proof of concept will soon be evaluated in a real device. Besides previous developments concerning PMR radios, the same method can be applied in every situation where cochannel interference make the RX works in its nonlinear region.

\section{ACKNOWLEDGMENT}

This work was funded in the scope of the FITNESS project (see [1]). The authors would like to thank all FITNESS partners and specifically the CEA for their cooperation.

\section{REFERENCES}

[1] FITNESS, "Fitness scope website," http://lisite.isep.fr/minarc/minarc-projects/fitness/, 2017, accessed: 2017-02-28.

[2] B. Razavi, RF Microelectronics (2Nd Edition) (Prentice Hall Communications Engineering and Emerging Technologies Series), 2nd ed. Upper Saddle River, NJ, USA: Prentice Hall Press, 2011.

[3] Q. Zou, M. Mikhemar, and A. H. Sayed, "Digital compensation of cross-modulation distortion in software-defined radios," IEEE Journal of Selected Topics in Signal Processing, vol. 3, no. 3, pp. 348-361, June 2009.

[4] P. Kenington, High-linearity RF Amplifier Design, ser. Artech House microwave library. Artech House, 2000. [Online]. Available: https://books.google.fr/books?id=qQhTAAAAMAAJ

[5] M. Valkama, A. S. hagh ghadam, L. Anttila, and M. Renfors, "Advanced digital signal processing techniques for compensation of nonlinear distortion in wideband multicarrier radio receivers," IEEE Transactions on Microwave Theory and Techniques, vol. 54, no. 6, pp. 2356-2366, June 2006.

[6] E. Rebeiz, A. S. H. Ghadam, M. Valkama, and D. Cabric, "Spectrum sensing under rf non-linearities: Performance analysis and dsp-enhanced receivers," IEEE Transactions on Signal Processing, vol. 63, no. 8, pp. 1950-1964, April 2015.

[7] R. Myer, "Automatic reduction of intermodulation products in high power linear amplifiers," Apr. 1 1986, uS Patent 4,580,105. [Online]. Available: https://www.google.com/patents/US4580105

[8] E. A. Keehr and A. Hajimiri, "Equalization of third-order intermodulation products in wideband direct conversion receivers," IEEE Journal of Solid-State Circuits, vol. 43, no. 12, pp. 28532867, Dec 2008.

[9] — "Successive regeneration and adaptive cancellation of higher order intermodulation products in rf receivers," IEEE Transactions on Microwave Theory and Techniques, vol. 59, no. 5, pp. 1379-1396, May 2011.

[10] N. Grollier, S. Houcke, and M. Pelissier, "Enhanced spectrally aware rf front end receiver under non-linearity," in 2018 IEEE International Conference on Communications (ICC), May 2018.

[11] N. Grollier and S. Houcke, "On carrier qpsk signal detector based on second order cyclic-moments," in 2018 IEEE Wireless Communications and Networking Conference (WCNC), April 2018, pp. 1-6. 